\title{
ON A THEOREM OF BRICKMAN-FILLMORE
}

\author{
ANTONIO HWANG
}

\begin{abstract}
Let $V$ be a finite dimensional vector space over an arbitrary field. We show that if $\operatorname{dim} V \leqslant 3$ and if $A, B$ and $C$ are pairwise commuting linear transformations on $V$ such that every subspace invariant for both $A$ and $B$ is also invariant for $C$, then $C$ is a polynomial in $A$ and $B$. (Brickman and Fillmore proved that if $B=0$ then this statement is true for any finite dimensional vector space $V$.) An example shows that this is not true for $\operatorname{dim} V>3$.
\end{abstract}

In [1] L. Brickman and P. A. Fillmore proved that if $A$ and $B$ are commuting linear transformations on a finite dimensional vector space over an arbitrary field and if every subspace invariant for $A$ is also invariant for $B$, then $B$ is a polynomial in $A$. Peter Fillmore suggested the following question (conveyed to me by Constantin Apostol):

If $A, B$ and $C$ are pairwise commuting linear transformations on a finite dimensional vector space $V$ over an arbitrary field and if every subspace invariant for both $A$ and $B$ is also invariant for $C$, then is $C$ a polynomial in $A$ and $B$ ?

We shall prove that the answer to this question is true if the dimension of $V$ is not more than 3 and false otherwise.

Suppose the dimension of $V$ is 2 . If $A$ has no nontrivial invariant subspace then $C$ is a polynomial in $A$ by the Brickman-Fillmore result. If $A$ is a scalar multiple of the identity then $C$ is a polynomial in $B$. Similar statements can also be made for $B$. Finally, if $A$ has a 1-dimensional eigenspace then $A, B$ and $C$ can be represented by upper triangular matrices relative to a fixed basis. By subtracting appropriate scalar multiples of the identity from $A, B$, and $C$, we may assume that:

$$
A=\left(\begin{array}{ll}
0 & a_{1} \\
0 & a_{2}
\end{array}\right), \quad B=\left(\begin{array}{ll}
0 & b_{1} \\
0 & b_{2}
\end{array}\right) \quad \text { and } C=\left(\begin{array}{cc}
0 & c_{1} \\
0 & c_{2}
\end{array}\right) .
$$

Since $A$ and $C$ commute we have $a_{1} c_{2}=c_{1} a_{2}$. Thus (i) $a_{1} \neq 0$ implies $\left(c_{1} / a_{1}\right) A=C$, (ii) $a_{2} \neq 0$ implies $\left(c_{2} / a_{2}\right) A=C$ and (iii) $a_{1}=a_{2}=0$ implies $C$ is a polynomial in $B$.

The proof for the case when the dimension of $V$ is 3 is obtained by considering the possible representations of $A$ given by the rational decomposition theorem. We omit the details.

Finally, let

Received by the editors November 7, 1974 and, in revised form, February 20, 1975.

AMS (MOS) subject classifications (1970). Primary 47A15.

Key words and phrases. Invariant subspace. 


$$
A=\left(\begin{array}{cccc}
0 & 0 & 0 & 0 \\
0 & 0 & 0 & 0 \\
1 & 0 & 0 & 0 \\
0 & 1 & 0 & 0
\end{array}\right), \quad B=\left(\begin{array}{cccc}
0 & 0 & 0 & 0 \\
0 & 0 & 0 & 0 \\
0 & 0 & 0 & 0 \\
1 & 0 & 0 & 0
\end{array}\right) \text { and } C=\left(\begin{array}{cccc}
0 & 0 & 0 & 0 \\
0 & 0 & 0 & 0 \\
1 & 0 & 0 & 0 \\
1 & 0 & 0 & 0
\end{array}\right)
$$

An easy computation shows that

$$
A B=B A=A C=C A=B C=C B=0 \text { and } A^{2}=B^{2}=C^{2}=0 .
$$

It follows from these that $C$ is not a polynomial in $A$ and $B$. To show that every subspace invariant under $A$ and $B$ is also invariant under $C$ it is sufficient to consider cyclic subspaces (that is, subspaces generated by the action of $A$ and $B$ on a single vector). An easy calculation shows that if $x$ is any vector, then $C x$ is a linear combination of $A x$ and $B x$. This example can be extended to the case $\operatorname{dim} V>4$ via direct sums.

\section{REFERENCE}

1. L. Brickman and P. A. Fillmore, The invariant subspace lattice of a linear transformation, Canad. J. Math. 19 (1967), 810-822. MR 35 \# 4242.

Department of Mathematics, University of Michigan, Ann Arbor, Michigan 48104

ibM Corporation, 2651 Strang Boulevard, Yorktown Heights, New York 10598

Current address: Apartment 2G, Woods End Circle, Peekskill, New York 10566 